\title{
Fluctuation-Induced Interactions between Rods on Membranes and Interfaces
}

\author{
Ramin Golestanian \\ Institute for Advanced Studies in Basic Sciences, Zanjan 45195-159, Iran \\ Mark Goulian* \\ Exxon Research and Engineering, Annandale, NJ 08801 \\ Mehran Kardar \\ Department of Physics, Massachusetts Institute of Technology, Cambridge, MA 02139
}

(November 6, 2018)

\begin{abstract}
We consider the interaction between two rods embedded in a fluctuating surface which is governed by either surface tension or rigidity. The modification of fluctuations by the rods leads to an attractive long-range interaction that falls off as $1 / R^{4}$ with their separation. The orientational dependence of the resulting interaction is non-trivial and may lead to interesting patterns of rod-like objects on such surfaces.
\end{abstract}

87.20, 82.65D, 34.20

Typeset using REVTEX

*Address after Aug. 31: Center for Studies in Physics and Biology, The Rockefeller University, 1230 York Avenue, New York, NY 10021. 
In both cell biology and in the design of biomolecular materials it is important to understand the interactions between inclusions in fluid membranes. The lipid bilayer defines the outer boundaries of both the cell and its interior organelles and vesicles. However, biomembranes play far more than simply a structural role; they also regulate and act as host for many bio- chemical and physical processes [1, 2]. For example, they control and regulate inter- and intra-cellular recognition and transport, adhesion, ion concentrations, and energy conversion. It is not surprising then, that biomembranes are far from homogeneous. In addition to the many different lipids that make up the bilayer, biomembranes contain a variety of proteins, glycolipids, and other macromolecules. Inclusions have also been incorporated in artificial membranes [2,3]. Such model-membrane systems have potential applications for targeted drug delivery and may also lead to novel "biologically inspired" materials, such as nano-scale pumps, templates, functionalized interfaces, and chemical reactors. Thus, for both biological and artificial membranes, it is important to understand how inclusions affect the physical properties of the membrane, and how the membrane in turn contributes to the interactions between inclusions.

There are a number of different forces which act within membranes [4]: van der Waals interactions fall off with separation $R$, as $1 / R^{6}$ at long distances; the Coulomb interaction is strongly screened under physiological conditions; hydration and structural forces are also short-ranged. There are additional interactions between inclusions which are mediated by the membrane; the inclusion disturbs the lipid bilayer and this disturbance propagates to neighboring inclusions (c.f. [2, [- 7] and references therein). When macroscopic thermal fluctuations are unimportant (we refer to this case as $T=0$ ), the resulting interactions tend to be short-ranged, falling off exponentially with a characteristic length corresponding to the distance over which the lipid disturbance "heals." For example, if in the region around an inclusion the membrane is forced to deviate from its preferred thickness $(\sim 40 \AA)$, then the resulting disturbance decays exponentially with a length comparable to this thickness [7].

If the membrane is to mediate long-ranged interactions, then in the long-distance limit it should be possible to neglect the membrane thickness and details concerning lipid structure. 
In this limit, the membrane is well-described by the elastic Hamiltonian [8],

$$
\mathcal{H}=\int \mathrm{d} S\left[\sigma+\frac{\kappa}{2} H^{2}+\bar{\kappa} K\right]
$$

where $\mathrm{d} S$ is the surface area element, and $H, K$ are the mean and Gaussian curvatures respectively. The elastic properties of the surface are described by the tension $\sigma$, and the bending rigidities $\kappa$ and $\bar{\kappa}$. A finite surface tension is the most important coupling in Eq.(11) and dominates the bending terms at long wavelengths. This is the case for films on a frame, interfaces at short distances, and possibly closed membranes in the presence of osmotic pressure differences between their interior and exterior. On the other hand, for closed bilayers in the absence of osmotic stress, as well as for microemulsions, the surface tension is effectively zero 9 [11]. In these cases, the energy cost of fluctuations is controlled by the rigidity terms. For simplicity we shall refer to surface tension dominated surfaces as films, and to rigidity controlled ones as membranes.

The long distance interactions between inclusions in a membrane were examined in Ref. [6]. It was shown that if the inclusions are asymmetric across the bilayer and impose a local curvature, Eq.(11) gives rise to a repulsive $(T=0)$ interaction that is long-ranged, falling off with distance as $1 / R^{4}$. The energy scale of the interaction is set by $\kappa$ and $\bar{\kappa}$. If thermal fluctuations of the membrane are included $(T \neq 0)$, on the other hand, there is a $1 / R^{4}$ interaction for generic inclusions, as long as the rigidity of the inclusion differs from that of the ambient membrane [6]. In particular, if the inclusions are much stiffer than the membrane, the potential has the form

$$
V(R)=-k_{B} T \frac{6 A^{2}}{\pi^{2} R^{4}}
$$

where $A$ is the area of each inclusion. (In the above formula, the result in Ref. [6] has been corrected by a factor of $1 / 2[15]$.) The interaction is attractive and independent of $\kappa$ and $\bar{\kappa}$; the energy scale is set by $k_{B} T$.

In this letter we consider the orientational interaction between rod-like inclusions due to thermal surface fluctuations $(T \neq 0)$. The rods are assumed to be much more rigid than 
the membrane so that they cannot move coherently with it; the only degrees of freedom for the rods are rigid translations and rotations while they remain attached to the surface. Conversely, the rods impose rigid boundary conditions on the surface, constraining its shape fluctuations. We will first give the results for the interaction energy and then briefly sketch their derivation; details of the calculations will be described in a separate paper [15]. Finally, we discuss some general aspects of this orientational interaction.

Consider a surface containing two rods of lengths $L_{1}$ and $L_{2}$ separated by a distance $R \gg L_{i}$. The rods make angles $\theta_{1}$ and $\theta_{2}$, respectively, with the line connecting their center of masses (see Fig. 1). For fluctuating films, we find an attractive fluctuation-induced interaction given by,

$$
V_{F}\left(R, \theta_{1}, \theta_{2}\right)=-\frac{k_{B} T}{128} \frac{L_{1}^{2} L_{2}^{2}}{R^{4}} \cos ^{2}\left[\theta_{1}+\theta_{2}\right]+O\left(1 / R^{6}\right)
$$

Note that this behavior is the square of a dipole-dipole interaction in two dimensions, with $L_{1}$ and $L_{2}$ as the dipole strengths. The fluctuation-induced interaction on a membrane is very similar and given by

$$
V_{M}\left(R, \theta_{1}, \theta_{2}\right)=-\frac{k_{B} T}{128} \frac{L_{1}^{2} L_{2}^{2}}{R^{4}} \cos ^{2}\left[2\left(\theta_{1}+\theta_{2}\right)\right]+O\left(1 / R^{6}\right) .
$$

In this case the interaction has the surprising property of being minimized for both parallel and perpendicular orientations of the rods. These interactions should have important effects in aligning asymmetric inclusions in biomembranes. Since orientational correlations are often easier to measure than forces, this result may also be useful in probing the fluctuationinduced interactions. Finally, this interaction may lead to novel two-dimensional structures for collections of rod-like molecules. In particular, the resemblance of the orientational part of the interaction to dipolar forces suggests that a suitable way to minimize the energy of a collection of rods is to form them into chains. (If the rods are not colinear, the interactions cannot be minimized simultaneously.) Such chain like structures are observed for ferromagnetic particles controlled by similar forces [12].

To obtain Eq.(4) we start with a thermally fluctuating planar membrane subject to the Hamiltonian in Eq.(1) (with $\sigma=0$ ). We assume that the size of the membrane $d$, is 
well below the persistence length $\xi$ [9]. In this limit, the membrane experiences only small fluctuations about a flat state. We may then parametrize the membrane surface with a height function $u(r)$, and approximate the full coordinate-invariant Hamiltonian in Eq.(11) by the Gaussian form $\mathcal{H}_{0}=\kappa / 2 \int \mathrm{d}^{2} r\left(\nabla^{2} u(r)\right)^{2}$. Now consider the situation depicted in Fig. 1, where two rigid, rod-like objects, are attached to the membrane. We shall represent the rods by narrow rectangles of lengths $L_{1}$ and $L_{2}$, and widths $\epsilon_{1}$ and $\epsilon_{2}$; ultimately taking the limit of $\epsilon_{i} \rightarrow 0$. The rods are constrained to fluctuate with the membrane but, due to their stiffness, can only be tilted or translated up and down rigidly. We can parametrize all possible configurations of the rods by

$$
\left.u(r)\right|_{r \in L_{i}}=a_{i}+\mathbf{b}_{i} \cdot \mathbf{r}, \quad \text { for } \quad i=1,2 \quad,
$$

where we have also used $L_{i}$ to denote the $i$ th rod.

To calculate the partition function, we follow a procedure similar to Ref. [6] and sum over all possible configurations of the membrane surface, weighted by the corresponding Boltzmann weight, and subject to the constraints imposed by the rods via Eq.(5). The constraints may be implemented with the aid of delta functions as in Ref. [13], leading to

$$
\mathcal{Z}=\int \mathcal{D} u(r) \prod_{i=1}^{2} \int \mathrm{d} a_{i} \mathrm{~d}^{2} b_{i} \prod_{r^{\prime} \in L_{i}} \delta\left(u\left(r^{\prime}\right)-a_{i}-\mathbf{b}_{i} \cdot \mathbf{r}^{\prime}\right) \exp \left[-\frac{\mathcal{H}_{0}}{k_{B} T}\right] .
$$

In Eq.(6) we have included only the leading term in an expansion in powers of $\mathbf{b}_{i}$, neglecting higher order terms from the projection of $L_{i}$ onto the $x-y$ plane, as well as from the integration measure for $\mathbf{b}_{i}$ on the sphere of unit normals. Since $\mathbf{b}_{i}$ controls the gradient of $u(r)$ at the boundary of $L_{i}$, the expansion in $\mathbf{b}_{i}$ is in the same spirit as the gradient expansion for $\mathcal{H}_{0}$. It can be shown that these higher order terms are irrelevant in the limit $d \ll \xi[15]$. Expressing the delta functions as functional integrals over auxiliary fields $k_{i}(r)$ defined on the rods, we obtain

$$
\begin{aligned}
\mathcal{Z}=\int \mathcal{D} u(r) \prod_{i=1}^{2} \int \mathrm{d} a_{i} \mathrm{~d}^{2} b_{i} \int_{L_{i}} \mathcal{D} k_{i}(r) \exp [ & -\frac{\kappa}{2 k_{B} T} \int \mathrm{d}^{2} r\left(\nabla^{2} u(r)\right)^{2} \\
& \left.+i \sum_{i=1}^{2} \int_{L_{i}} \mathrm{~d}^{2} r_{i} k_{i}\left(r_{i}\right)\left(u\left(r_{i}\right)-a_{i}-\mathbf{b}_{i} \cdot \mathbf{r}_{i}\right)\right]
\end{aligned}
$$


Integrating out $u(r), a_{i}$, and $\mathbf{b}_{i}$, then gives (throughout we drop irrelevant multiplicative constants in $\mathcal{Z}$ )

$$
\begin{aligned}
\mathcal{Z}=\prod_{i} \int \mathcal{D} k_{i}(r) \delta\left(\int_{L_{i}} \mathrm{~d}^{2} r_{i} k_{i}\left(r_{i}\right)\right) \delta\left(\int_{L_{i}} \mathrm{~d}^{2} r_{i} \mathbf{r} k_{i}\left(r_{i}\right)\right) \times \\
\exp \left[-\frac{k_{B} T}{2 \kappa} \sum_{i, j=1}^{2} \int_{L_{i}} \mathrm{~d}^{2} r_{i} \int_{L_{j}} \mathrm{~d}^{2} r_{j} k_{i}\left(r_{i}\right) G\left(\mathbf{r}_{i}-\mathbf{r}_{j}\right) k_{j}\left(r_{j}\right)\right],
\end{aligned}
$$

where $G\left(\mathbf{r}-\mathbf{r}^{\prime}\right)=(1 / 8 \pi)\left|\mathbf{r}-\mathbf{r}^{\prime}\right|^{2} \ln \left|\mathbf{r}-\mathbf{r}^{\prime}\right|$.

Equation (8) is analogous to the partition function for a pair of plasmas confined to the interior of rods $L_{1}$ and $L_{2}$. The delta functions impose the constraints that the net charge and dipole moments vanish within each rod. When the distance $R$ between rods is

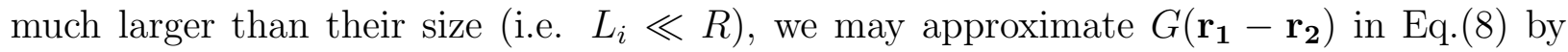
a multipole expansion and keep only the leading term, which comes from the quadrupole moments $Q_{a b}^{(i)} \equiv \int_{L_{i}} \mathrm{~d}^{2} r r_{a} r_{b} k_{i}(r)$, to get

$$
\begin{aligned}
\mathcal{Z}= & \prod_{i} \int \mathcal{D} k_{i}(r) \int \mathrm{d} \mathbf{Q}^{(i)} \mathrm{d} a_{i} \mathrm{~d}^{2} b_{i} d \mathbf{g}^{(i)} \exp \left\{-\frac{k_{B} T}{2 \kappa} \sum_{i} \int_{L_{i}} \mathrm{~d}^{2} r \mathrm{~d}^{2} r^{\prime} k_{i}(r) G\left(\mathbf{r}-\mathbf{r}^{\prime}\right) k_{i}\left(r^{\prime}\right)\right. \\
& \left.-i \sum_{i} \int_{L_{i}} \mathrm{~d}^{2} r k_{i}(r)\left[a_{i}+\mathbf{b}_{i} \cdot \mathbf{r}+\mathbf{r} \cdot \mathbf{g}^{(i)} \cdot \mathbf{r}\right]+i \sum_{i} g_{a b}^{(i)} Q_{a b}^{(i)}-\frac{k_{B} T}{2 \kappa} v\left[\mathbf{Q}^{(1)}, \mathbf{Q}^{(2)}\right]\right\},
\end{aligned}
$$

with the quadrupole-quadrupole interaction $v\left[\mathbf{Q}^{(1)}, \mathbf{Q}^{(2)}\right]$ the same as in Ref. [6].

We first isolate the integration over $k_{1}(r)$ in Eq.(9),

$$
\begin{aligned}
I_{1} \equiv \int \mathcal{D} k_{1}(r) \mathrm{d} a_{1} \mathrm{~d}^{2} b_{1} \exp \{ & -\frac{k_{B} T}{2 \kappa} \int_{L_{1}} \mathrm{~d}^{2} r \mathrm{~d}^{2} r^{\prime} k_{1}(r) G\left(\mathbf{r}-\mathbf{r}^{\prime}\right) k_{1}\left(r^{\prime}\right) \\
& \left.-i \int_{L_{1}} \mathrm{~d}^{2} r k_{1}(r)\left[a_{1}+\mathbf{b}_{1} \cdot \mathbf{r}+\mathbf{r} \cdot \mathbf{g}^{(1)} \cdot \mathbf{r}\right]\right\} .
\end{aligned}
$$

To perform the above integration, the Green's function $G\left(\mathbf{r}-\mathbf{r}^{\prime}\right)$ has to be inverted in the finite region $L_{1}$. To do this, we introduce an auxiliary field $h(\mathbf{r})$ over the whole plane (indicated by $\mathrm{IR}^{2}$ ) and write

$$
I_{1}=\int \mathcal{D} h(r) \mathrm{d} a_{1} \mathrm{~d}^{2} b_{1} \exp \left[-\frac{\kappa}{2 k_{B} T} \int_{\mathrm{IR}^{2}} \mathrm{~d}^{2} r\left(\nabla^{2} h(r)\right)^{2}\right] \prod_{r^{\prime} \in L_{1}} \delta\left(h\left(r^{\prime}\right)-a_{1}-\mathbf{b}_{1} \cdot \mathbf{r}-\mathbf{r} \cdot \mathbf{g}^{(1)} \cdot \mathbf{r}\right) .
$$

Integration over $h(r), a_{1}, \mathbf{b}_{1}$ gives (dropping a multiplicative constant) [15] 


$$
I_{1}=\exp \left\{-\frac{\kappa}{k_{B} T}\left[2 \epsilon_{1} L_{1}\left(g_{a a}^{(1)}\right)^{2}+\pi\left(L_{1} g_{x y}^{(1)}\right)^{2}\right]\right\}
$$

The result of the $k_{2}(r)$ integration in Eq.(9) is similar, with the index 2 replacing 1, and with the coordinate axis appropriately rotated to align with the second rod. The overall expression for the partition function now reads

$$
\begin{aligned}
\mathcal{Z}=\prod_{i=1}^{2} \int \mathrm{d} \mathbf{Q}^{(i)} \mathrm{d} \mathbf{g}^{(i)} \exp \left\{-\frac{\pi \kappa}{k_{B} T}\left[\left(L_{1} g_{x^{\prime} y^{\prime}}^{(1)}\right)^{2}+\left(L_{2} g_{x^{\prime \prime} y^{\prime \prime}}^{(2)}\right)^{2}\right]\right\} \times \\
\exp \left\{-i \sum_{i} g_{a b}^{(i)} Q_{a b}^{(i)}-\frac{k_{B} T}{2 \kappa} v\left[\mathbf{Q}^{(1)}, \mathbf{Q}^{(2)}\right]\right\}
\end{aligned}
$$

where we have set the widths of the rods to zero $\left(\epsilon_{i} \rightarrow 0\right)$. The primed indices $x^{\prime}, y^{\prime}, x^{\prime \prime}, y^{\prime \prime}$ indicate that the corresponding components are with respect to the coordinate frames where $L_{1} \| y^{\prime}$ and $L_{2} \| y^{\prime \prime}$. We define an un-primed coordinate system such that the $x$-axis is parallel to $\hat{R}$ and the two rods make angles of $\theta_{1}$ and $\theta_{2}$ with respect to the $x$-axis as in Fig. 1 . After performing the remaining integrations, which are cumbersome but straightforward, we end up with the $\left(R, \theta_{1}, \theta_{2}\right)$ dependent part of the free energy given in Eq.(4).

The calculation for films is similar. In this case, the bending rigidity is set to zero in Eq.(11). We restrict to sufficiently large surface tensions such that $\sigma a^{2} / k_{B} T \gg 1$, with $a$ some microscopic length. We can then approximate Eq.(1) by the Gaussian Hamiltonian $\mathcal{H}_{0}=\sigma / 2 \int \mathrm{d}^{2} r(\nabla u(r))^{2}$. Computing the partition function along similar lines, we find that the leading behavior results from fluctuating dipoles instead of quadrupoles. However, the calculation is complicated by the fact that the dependence on $\mathbf{b}_{i}$ is more complex than in the case of the membrane. To extract a simple answer, we assume $\sigma L^{4} / R^{2} \ll k_{B} T$, and find the result in Eq.(3).

The interactions in Eqs.(3) and (国) have a number of interesting properties. First, their magnitude is set by $k_{B} T$ and is independent of the tension and rigidity coefficients $\sigma$ and $\kappa$. Thus the effect persists even for rather stiff membranes with $\kappa \gg k_{B} T$; as long as the inclusions are more rigid than the embedding surface. Second, the interaction falls off with distance as $1 / R^{4}$. This is a general feature of fluctuation-induced forces, including the van der Waals interaction, which in $d$ dimensions falls off as $1 / R^{2 d}$. (Surprisingly, a recent 
calculation of similar orientational forces for a polymer on a flexible surface [14] finds different asymptotic decays for films and membranes.) Since the direct van der Waals interactions fall off as $1 / R^{6}$, the forces mediated through the two-dimensional surface will always dominate asymptotically. Finally, the most interesting aspect is the orientational dependence of the force: The angular dependence is a squared dipolar interaction for inclusions on a film, and a squared quadrupolar interaction on a membrane. By comparison, an additive interaction, between any two infinitesimal line elements, leads to an orientation dependence of $\cos 2 \theta_{1}+$ $\cos 2 \theta_{2}$. This angular dependence is completely different, and minimized when the two rods are parallel to their axis of separation. Presumably both interactions are present for rods of finite thickness; the additive interaction is proportional to $(L \epsilon)^{2}$, where $\epsilon$ is the thickness. The previously calculated interactions are thus larger by a factor proportional to $(L / \epsilon)^{2}$ and should dominate for thin rods.

MK and MG acknowledge the hospitality of the ITP at Santa Barbara where this work was initiated (supported by NSF Grant No. PHY-89-04035). The work at MIT is supported by the NSF grant DMR-93-03667. RG acknowledges support from the Institute for Advanced Studies in Basic Sciences at Gava Zang, Zanjan, Iran. 


\section{REFERENCES}

[1] B. Alberts, J. Lewis, M. Raff, K. Roberts and J.D. Watson, Molecular Biology of the Cell, Garland, New York 1994.

[2] R.B. Gennis, Biomembranes, Molecular Structure and Function, Springer-Verlag, New York 1989.

[3] Y. Shen, C.R. Safinya, K.J. Rothschild, K.S. Liang, and A.F. Ruppert, Nature, 366 (1993) 48 (and references therein).

[4] J. Israelachvili, Intermolecular and Surface Forces, Academic Press, San Diego 1992.

[5] O.G. Mouritsen and M. Bloom, Annu. Rev. Biophys. Biomol. Struct., 22 (1993) 145.

[6] M. Goulian , R. Bruinsma, and P. Pincus, Europhys. Lett., 22 (1993) 145; Erratum in Europhys. Lett. 23 (1993) 155.

[7] N. Dan, P. Pincus, and S.A. Safran, Langmuir, 9 (1993) 2768.

[8] P.B. Canham, J. Theor. Biol., 26 (1970) 61; W. Helfrich, Z. Naturforsch., 28c (1973) 693.

[9] P.G. de Gennes and C. Taupin, J. Phys. Chem., 86 (1982) 2294.

[10] F. Brochard and J.F. Lennon, J. de Phys., 36 (1975) 1035.

[11] F. David and S. Leibler, J. de Phys. II, 1 (1991) 959.

[12] R.E. Rosensweig, Ferrohydrodynamics, Cambridge, New York 1985.

[13] H. Li and M. Kardar, Phys. Rev. Lett. 67, 3275 (1991); Phys. Rev. A46, 6490 (1992).

[14] R. Podgornik, preprint, cond-mat/9503146 (1995).

[15] R. Golestanian, M. Goulian, M. Kardar, in preparation. 


\section{FIGURES}

FIG. 1. The $i$ th rod has length $L_{i}$, width $\epsilon_{i}$, and makes an angle $\theta_{i}$ with the line joining the centers of the two rods. In the text, the distance between rods $R$ is taken to be much larger than $L_{1}$ and $L_{2}$. 


$$
2 \pi
$$

